\title{
Gastrointestinal Bleeding During Index Hospitalization for Mechanical Circulatory Support Devices Implantation: Is the Squeeze Worth the Ooze?
}

\author{
Darius A. Jahann' ${ }^{1}$ Vanessa M. Shami ${ }^{1}$
}

Published online: 26 November 2016

(c) Springer Science+Business Media New York 2016

Mechanical circulatory support devices, which include left ventricular assist devices (LVADs) and intra-aortic balloon pumps (IABPs), have been used increasingly over the past decade. LVADs sustain patients with severe left ventricular dysfunction who are being evaluated for or await cardiac transplantation, as a destination therapy, or a bridge to recovery of cardiac function [1]. Their increasing popularity in the past decade is partially attributable to remarkable advances in LVAD technology. First-generation devices, which functioned by a mechanism of pulsatile blood flow, were beset by poor durability and high rates of infection and thrombosis, encouraging the development of smaller, continuous-flow LVADs which involve rotarypump technology to provide blood flow with reduced pulsatility. These latter devices have improved the probability of survival free from stroke and device failure at 2 years compared with the first-generation device [2]. Nevertheless, the increasing use of this technology has been accompanied by a notable rate of gastrointestinal bleeding (GIB) with a recent meta-analysis estimating the pooled prevalence of GIB in LVAD patients to be an astounding 23\% [3].

Meanwhile, the IABP is the single most widely used circulatory assist device. In contrast to available data for GIB in LVADs, the data for GIB following IABP placement are limited to a small study of 62 IABP patients which estimated the risk of GIB following IABP implantation at $8 \%$ [4]. With the widespread use of these

Vanessa M. Shami

VMS4E@virginia.edu

1 Division of Gastroenterology and Hepatology, University of Virginia Health System, PO BOX 800708, Charlottesville, VA 22908, USA mechanical circulatory devices, more data on the incidence of adverse events such as bleeding are needed.

In this issue of Digestive Diseases and Sciences, Abbas et al. [5] published a retrospective study describing the prevalence and predictors of GIB during the index hospitalization of patients who underwent mechanical circulatory support device implantation. The authors were primarily interested in LVADs, but also sought to investigate the risk of GIB following IABP placement. Using the Nationwide Inpatient Sample, the authors identified patients with the discharge diagnosis codes for heart failure and procedure codes for LVAD, IABP, or OHT (orthotopic heart transplant). A total of 87,462 patients were included for analysis, among which $87 \%$ received an IABP, $6 \%$ received $\mathrm{LVAD}$, and 5\% had OHT. The primary outcome measure was GIB during the index hospitalization during which the mechanical circulatory support device was implanted. Univariate analysis determined that patients who underwent LVAD plus IABP implantation had the highest prevalence of GIB at $10.3 \%$, followed by patients "bridged" to heart transplant with a device, LVAD patients, IABP implantation, and OHT recipients who had a prevalence of $8.9,8.6,5.8$, and $3.3 \%$, respectively. The source of GIB was more likely to be due to a lower etiology in LVAD and OHT recipients, 53.1 and $57.7 \%$, whereas an upper source was the culprit in approximately $30 \%$ of cases. The most common hindgut etiology in LVAD recipients was "uncategorized," followed by diverticulosis. OHT recipients most often experienced lower GIB due to arteriovenous malformation or anorectal pathologies such as hemorrhoids or anal fissure. The most common etiology of lower GIB in LVAD and OHT recipients was gastroduodeno-jejunal mucosal erosive disease such as ulceration or inflammation in approximately $15 \%$. Despite hindgut bleeding etiologies being more common, upper endoscopy 
was performed more frequently than was lower endoscopy. Multivariate analysis identified that LVAD recipients had a twofold increase in developing a GIB during index hospitalization when compared to OHT and IABP recipients in two separate analyses. Prolonged length of stay, acute kidney injury requiring dialysis, moderate-severe liver disease, disseminated intravascular coagulation, sepsis, and mechanical ventilation were all independently associated with GIB. The authors also identified risk factors not previously recognized, which were associated with a higher prevalence of GIB in this patient population-advanced age, female gender, Asian, and African-American race. Lastly, though the univariate analysis noted a higher prevalence of GIB among IABP recipients, there was no difference when multivariate analysis was used after controlling for demographics, medical comorbidities, and inpatient incidents such as mechanical ventilation and sepsis.

The strength of this article lies in its analysis of a considerably large patient population-87,462, of which 4869 were LVAD recipients, accomplished by using the Nationwide Inpatient Sample, the largest all-payer inpatient care database in the USA. This enables the authors to analyze a massive dataset, substantially larger than any previous study on this matter. All prior publications addressing GIB subsequent to LVAD placement included a combined total of 1697 patients [3].

The use of a large database, however, is not without its limits since much of the data were derived from the International Classification of Diseases (ICD)-9 codes, a factor acknowledged by the authors and evident in the data regarding etiology of GIB. The code assignment process is complicated, with various steps and participants, introducing opportunities for error. Though prior data on diagnosis codes for patients with upper GI hemorrhage have demonstrated reasonable accuracy (85-95\%), this study that relies heavily on ICD-9 codes can expect some degree of bias from those errors [6]. For instance, in contrast to other studies, the authors observed that lower sources were more common than upper sources of GIB. This could be partly due to a large proportion of GIB that was categorized as neither upper nor lower (19\%) as well as the percentage of lower GIB that was uncategorized (18\%).

Despite available epidemiological data regarding bleeding after mechanical circulatory device implantation, the underlying pathogenesis of GIB in these patients is not fully understood. The normal cardiac physiologic state is pulsatile blood flow, and though newer-generation LVADs have their aforementioned advantages, their continuous, non-pulsatile flow increases the risk of bleeding [7]. The proclivity for bleeding in a state of reduced pulsatility created by continuous-flow LVADs has not been completely elucidated but may be related to systemic and local factors including formation of arteriovenous malformations, acquired von Willebrand disorder, and mucosal ischemia [8]. Though the type of LVAD flow was not available in the analyzed data, the LVAD population in Abbas et al. [5] likely represented a significant majority of newer-generation non-pulsatile flow devices according to cited registry data in their Discussion; conversely, IABPs work to circulate blood via pulsatile flow. Thus, in their comparison of LVAD and IABP patients for multivariate analysis, the authors are isolating the underlying mechanism of flow as a predictor of the primary outcome, in addition to mitigating the effect of anticoagulation use present in both groups. Regarding risk of GIB among IABP recipients, a higher prevalence was not appreciated when multivariate analysis was performed, reinforcing the concept that a state of artificial pulsatile blood flow in and of itself may not be a risk factor for bleeding in these patients.

Overall, this study is a notable addition to the available literature on GIB following mechanical circulatory device implantation. By using the Nationwide Inpatient Sample, Abbas et al. included more patients than all prior studies combined and thoughtfully described the prevalence of GIB during index hospitalization following LVAD implantation and IABP implantation, the latter for which scant prior data were available. Furthermore, the authors described novel risk factors associated with a higher prevalence of GIB in this patient population-advanced age, female gender, Asian, and African-American race, enabling more effective risk stratification of patients when weighing the risk/benefit of device implantation. Future studies should provide more data on particular endoscopic interventions and their effect on patient outcomes. Unfortunately for many in this patient population, there are not any other viable options, and the squeeze is very well worth the potential ooze.

\section{Compliance with ethical standards}

Conflict of interest None.

\section{References}

1. Ammirati E, Oliva F, Cannata A, et al. Current indications for heart transplantation and left ventricular assist device: a practical point of view. Eur J Intern Med. 2014;25:422-429.

2. Slaughter MS, Rogers JG, Milano CA, et al. Advanced heart failure treated with continuous-flow left ventricular assist device. N Engl J Med. 2009;3:2241-2251.

3. Draper KV, Huang RJ, Gerson LB. GI bleeding in patients with continuous-flow left ventricular assist devices: a systematic review and meta-analysis. Gastrointest Endosc. 2014;80:435-446.e1.

4. Boudoulas KD, Pederzolli A, Saini U, et al. Comparison of Impella and intra-aortic balloon pump in high-risk percutaneous coronary intervention: vascular complications and incidence of bleeding. Acute Card Care. 2012;14:120-124. 
5. Abbas A, Mahmoud A, Ahmed M, Aranda J, Klodell CT, Draganov PV. Gastrointestinal bleeding during the index hospitalization for mechanical circulatory support devices implantation, a nationwide perspective. Dig Dis Sci. 2016. doi:10.1007/s10620016-4271-6.

6. Cooper GS, Chak A, Lloyd LE, Yurchick PJ, Harper DL, Rosenthal GE. The accuracy of diagnosis and procedural codes for patients with upper GI hemorrhage. Gastrointest Endosc. 2000;1:423-426.
7. Kushnir VM, Sharma S, Ewald GA, Seccombe J, Novak E, Wang I-W, et al. Evaluation of GI bleeding after implantation of left ventricular assist device. Gastrointest Endosc. 2012;75:973-979.

8. Uriel N, Pak S-W, Jorde UP, et al. Acquired von Willebrand syndrome after continuous-flow mechanical device support contributes to a high prevalence of bleeding during long-term support and at the time of transplantation. $J$ Am Coll Cardiol. 2010;5:1207-1213. 